\title{
Robust Adaptive Beamforming Applied to Planar Array
}

\author{
SONG Hai-yan \\ School of Electrical and Information Engineering \\ Heilongjiang Institute of Technology \\ Harbin, China \\ songhaiyan0508@hrbeu.edu.cn \\ SHI Jie* \\ Science and Technology on Underwater Acoustic Laboratory \\ Harbin Engineering University \\ Harbin, China \\ shijie080428@yahoo.com.cn \\ LIU Bo-sheng \\ College of Underwater Acoustic Engineering \\ Harbin Engineering University \\ Harbin, China \\ TIAN Ye \\ Engineering Training Centre \\ Harbin Engineering University \\ Harbin, China \\ YE Jun \\ 65426 army \\ Harbin, China
}

\begin{abstract}
Capon beamforming has better resolution and interference rejection capability. However, its performance will seriously degrade due to noise, array steering vector error, and other factors. In this paper, a robust Capon beamforming applied to a planar array is described. It is shown that the proposed method is the natural extension of the original Vector Optimization Robust Beamforming algorithm to the case of a planar array, and can be reformulated as a convex secondorder cone program and solved by SEDUMI. Computer simulation has shown that the proposed method has better performance than other conventional methods, such as narrower main lobe and lower side lobe.
\end{abstract}

Keywords- robust, Capon beamforming, planar array, second-order cone program, Vector Optimization

\section{INTRODUCTION}

In many practical array signal processing problems, beamforming is an important topic with applications in sonar, radar, communication, and other research areas. In general, beamforming includes Conventional Beamforming

*Corresponding Author

Project supported by the Specialized Research Fund for the Doctoral Program of Higher Education of China (Grant No. 20122304120011), the Fundamental Research Funds for the Central Universities (Grant No. HEUCFR1119) and the Research Fund for the Doctor of Heilongjiang Institute of Technology (Grant No. 2012BJ23)
(CBF) and Standard Capon Beamforming (SCB), and the latter has a better resolution and much better interference rejection capability than the former ${ }^{[1]}$. However, under the conditions of noise, array steering vector error, and other factors, SCB can no longer perform well, and its performance will become worse than $\mathrm{CBF}^{[2]}$.

There have been several approaches to such problems including Linearly Constrained Minimum Variance Beamformer (LCMV), Diagonal Loading (DL) and so on ${ }^{[3]}$. Recently, a robust Capon beamformer is proposed respectively by Li and Vorobyov, which can account for the problem of the uncertain steering vectors and be solved by second-order cone program (SOCP) efficiently ${ }^{[4-5]}$. In addition, the robust SCB algorithm has been used for localization and identification of moving sound sources ${ }^{[6]}$. In an attempt to overcome a substantial degradation of the SCB performance in situations of small training sample size and imprecise knowledge of the Signal Of Interest (SOI) steering vector, a new algorithm called Vector Optimization Robust Beamforming (VORB) is proposed by Song, which is simpler and highly robust against the array steering vector error ${ }^{[7-8]}$

However, the existing robust beamforming approaches are almost based on a horizontal or vertical array, and can only measure the incidence angle or the elevation angle. In order to accurately obtain both incidence angle and elevation angle at the same time, the present work in this paper extends the VORB algorithm to a vertical planar array. Briefly, the proposed method fully exploits the property of 
VORB and follows the same idea as before except on the basis of a vertical planar array. Finally, it is shown that the method can be reformulated as a SOCP and solved by SEDUMI $^{[9]}$. Computer simulation has shown that the proposed method has better performance than CBF and SCB, such as narrower main lobe and lower side lobe.

The content organization is as follows: Section 1 introduces some background of robust capon beamforming. Section 2 presents the planar array signal model and describes the formulation of the proposed method. Section 3 presents the computer simulation results, and the spatial spectrums of our proposed method are analyzed. The conclusions are summarized in Section 4.

\section{VECTOR OPTIMIZATION ROBUST ADAPTIVE BEAMFORMING BASED ON PLANAR ARRAY}

\section{A. Planar array signal model}

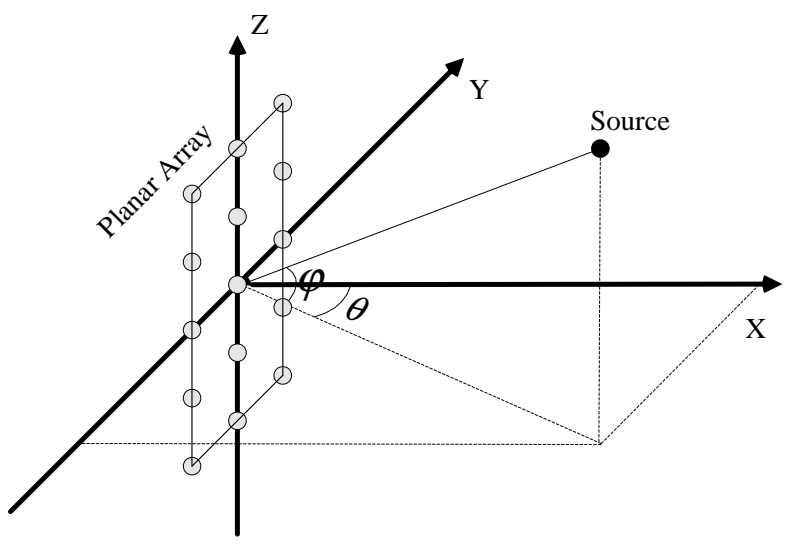

Figure 1. The sketch map of planar array signal model

As shown in Fig.1, consider a planar vertical array of $N$ equally spaced hydrophones, and set the central hydrophone (reference element) as the origin, so the coordinate of each element can be expressed as $\left(y_{n}, z_{n}\right),(n=1,2, \cdots, N)$. Assume there exist $M$ sources in the far field of the array, and the $m$ th source has the incidence angle $\theta_{m}$, elevation angle $\varphi_{m}$. Based on above assumptions, the time delay for the $m$ th source from the $n$th element to the reference element can be written as:

$$
\tau_{n m}=\frac{1}{c}\left(y_{n} \sin \theta_{m} \cos \varphi_{m}+z_{n} \sin \varphi_{m}\right)
$$

Where $c$ is the sound propagation velocity.

So the received signal can be given by:

$$
X(t)=A(\theta, \varphi) S(t)+N(t)
$$

Where $\boldsymbol{X}(t)=\left[x_{1}(t), x_{2}(t), \cdots, x_{N}(t)\right]^{\mathrm{T}} \quad$ denotes the $N \times 1$ dimension received signal vector, $\boldsymbol{S}(t)=\left[s_{1}(t), s_{2}(t), \cdots, s_{M}(t)\right]^{\mathrm{T}}$ denotes the $M \times 1$ dimension transmit signal vector, $N(t)=\left[n_{1}(t), n_{2}(t), \cdots, n_{N}(t)\right]^{\mathrm{T}} \quad$ denotes the $N \times 1$ dimension noise vector, and $A(\theta, \varphi)=\left[\boldsymbol{a}\left(\theta_{1}, \varphi_{1}\right), \boldsymbol{a}\left(\theta_{2}, \varphi_{2}\right), \cdots, \boldsymbol{a}\left(\theta_{M}, \varphi_{M}\right)\right]$ denotes the $N \times M$ dimension steering vector matrix.

The steering vector $\boldsymbol{a}\left(\theta_{m}, \varphi_{m}\right) \quad(m=1,2, \cdots, M)$ can be represented as:

$$
\boldsymbol{a}\left(\theta_{m}, \varphi_{m}\right)=\left[e^{-j \omega \tau_{1 m}}, e^{-j \omega \tau_{2 m}}, \cdots, e^{-j \omega \tau_{N m}}\right]^{\mathrm{T}}
$$

\section{B. Formulation}

Below, we provide the DOA estimation formulation based on the planar array following exactly the same procedure in Ref.[4-5]. In practical applications, there must exist a distortion of the steering vector matrix $A(\theta, \varphi)$, (for convenient, we represent the distortion as $\boldsymbol{\Delta}$ ), and we can bound the norm of $\boldsymbol{\Delta}$ by a certain constant $\gamma>0$, that is

$$
\|\boldsymbol{\Delta}\|_{2} \leq \gamma
$$

So the actual steering vector matrix $\tilde{A}(\theta, \varphi)$ belongs to the following set

$$
\boldsymbol{E}((\theta, \varphi) \mid \gamma)=\left\{\tilde{A}(\theta, \varphi) \mid \tilde{A}(\theta, \varphi)=A(\theta, \varphi)+\Delta,\|\Delta\|_{2} \leq \gamma\right\}
$$

Where $\|\cdot\|_{2}$ denotes the 2-norm, and $\boldsymbol{E}((\theta, \varphi) \mid \gamma)$ is an uncertainty set whose elements represent the actual possible steering vector matrix.

Let's impose a constraint on the absolute value of the array response, that is, the value should be larger than or equal to one for all vectors that belong to $\boldsymbol{E}((\theta, \varphi) \mid \gamma)$

$$
\left|\boldsymbol{w}^{\mathrm{H}} \tilde{\boldsymbol{A}}(\theta, \varphi)\right| \geq 1 \text {, for all } \tilde{\boldsymbol{A}}(\theta, \varphi) \in \boldsymbol{E}((\theta, \varphi) \mid \gamma)
$$

Where $\boldsymbol{w}$ is the weight vector.

Make explicit use of the idea of VORB and the planar array signal model, then we can reformulate the optimization problem as

$$
\left\{\begin{aligned}
& \min _{\boldsymbol{w}}\left(\text { w.r.t. } \boldsymbol{R}_{+}^{2}\right) \quad\left(\|\boldsymbol{U} \boldsymbol{w}\|_{2},\|\boldsymbol{w}\|_{2}\right) \\
\text { s.t. } & \boldsymbol{w}^{\mathrm{H}} \boldsymbol{A}(\theta, \varphi) \geq \gamma\|\boldsymbol{w}\|_{2}+1, \quad \operatorname{Im}\left\{\boldsymbol{w}^{\mathrm{H}} \boldsymbol{A}(\theta, \varphi)\right\}=0
\end{aligned}\right.
$$

Where w.r.t. represents "with respect to", $\boldsymbol{R}_{+}^{2}$ means that we should discuss $\|\boldsymbol{U} \boldsymbol{w}\|_{2}$ and $\|\boldsymbol{w}\|_{2}$ in the set of positive numbers, and $\boldsymbol{U}$ is given by the Cholesky factorization $\boldsymbol{R}=\boldsymbol{U}^{\mathrm{H}} \boldsymbol{U}$.

According to Ref.[8], equ.(7) can be implemented by SOCP or Lagrange multiplier method, and solved by SEDUMI[9].

\section{COMPUTER SIMULATIONS}

Simulation condition: The number of the array elements is 35 ( there are 5 rows along the coordinate axis $y$ and 7 columns along the coordinate axis $\mathrm{z}$ ), the source frequency is $200 \mathrm{kHz}$, the element spacing is half-wavelength, the incidence angle and the elevation angle are respectively $\theta=-1^{\circ}$ and $\varphi=1^{\circ}$, SNR is $20 \mathrm{~dB}$, the array steering vector error is $-5 \mathrm{~dB}$, which is defined as: 


$$
10 \log _{10}\left(\frac{\|\Delta\|_{\mathrm{F}}^{2}}{\|\tilde{\boldsymbol{A}}(\theta, \varphi)\|_{\mathrm{F}}^{2}}\right)
$$

Fig.2, Fig.3, and Fig.4 respectively show the spatial spectrums by CBF, MVDR, and VORB. Note that under the condition of the noise and the array steering vector error, the VORB has better performance than CBF and MVDR, such as the narrower main lobe and the lower side lobe. It is obvious from the simulation figures that VORB can suppress the side lobe to - $20 \mathrm{~dB}$ while MVDR only $-6 \mathrm{~dB}$, so VORB can highly improve the robustness against the noise and the array model error.

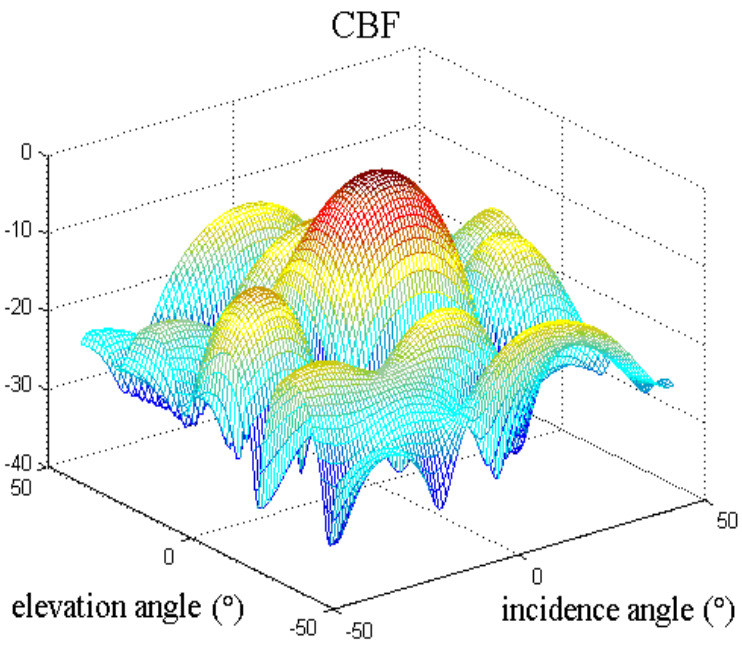

(a) Three-dimension graph $\mathrm{CBF}$

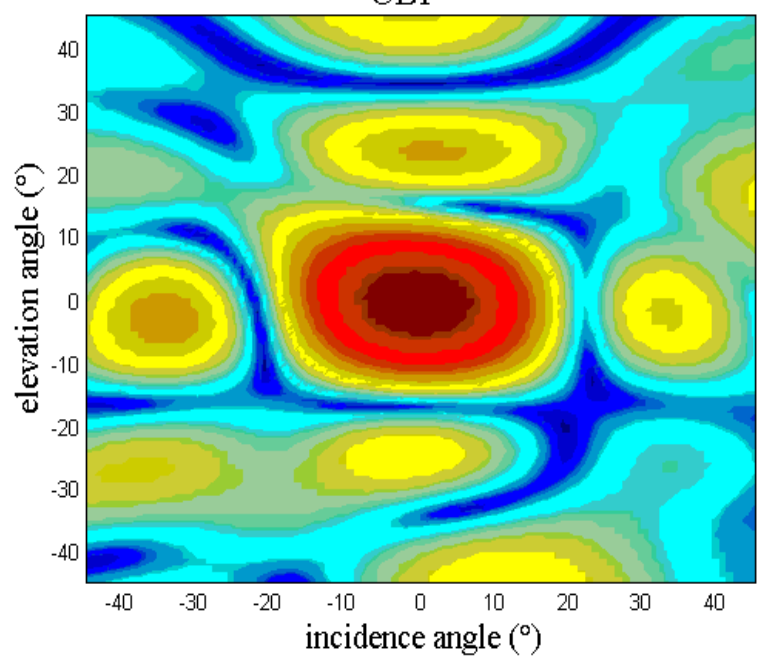

(b) Top-view graph

Figure 2. The spatial spectrum for CBF

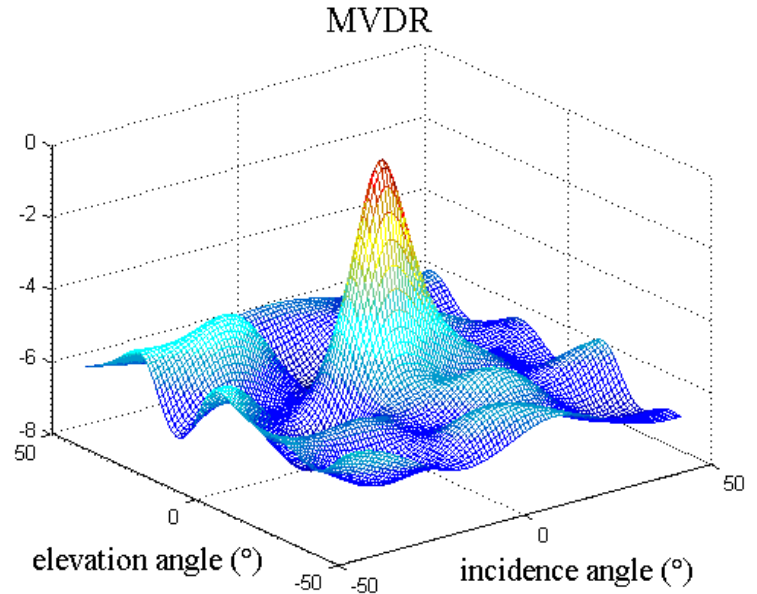

(a) Three-dimension graph

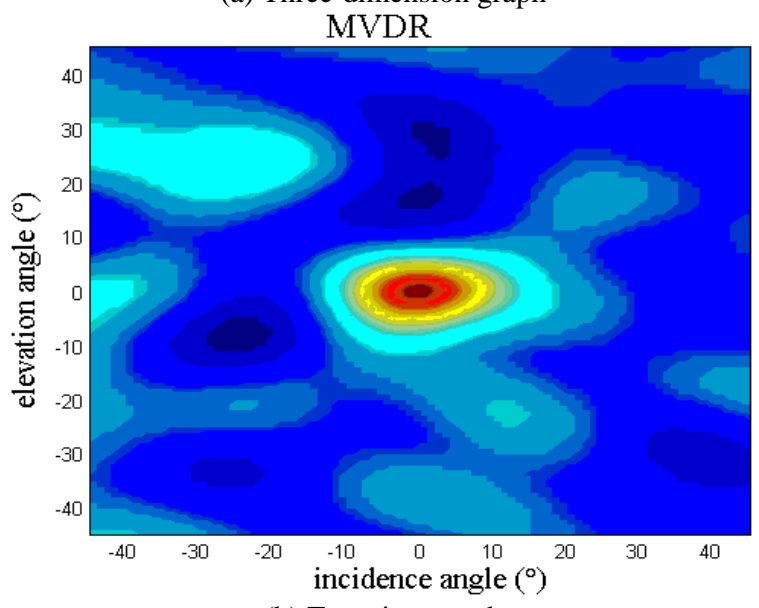

(b) Top-view graph

Figure 3. The spatial spectrum for MVDR

Proposed method

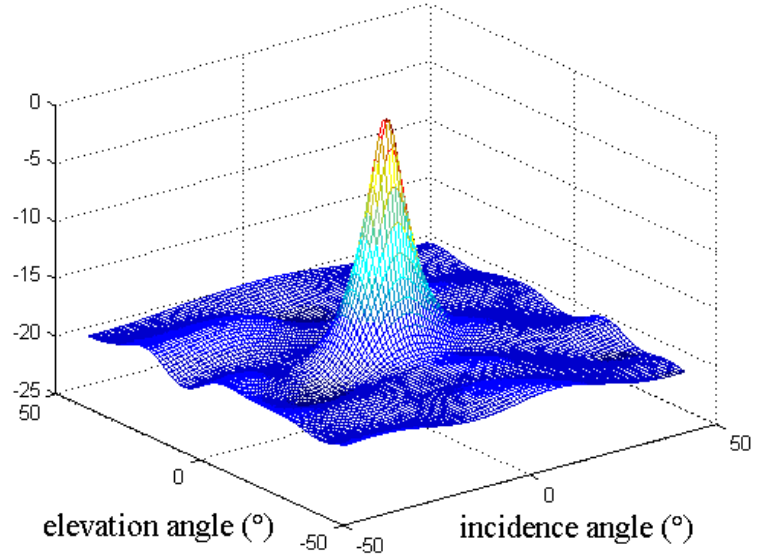

(a) Three-dimension graph 


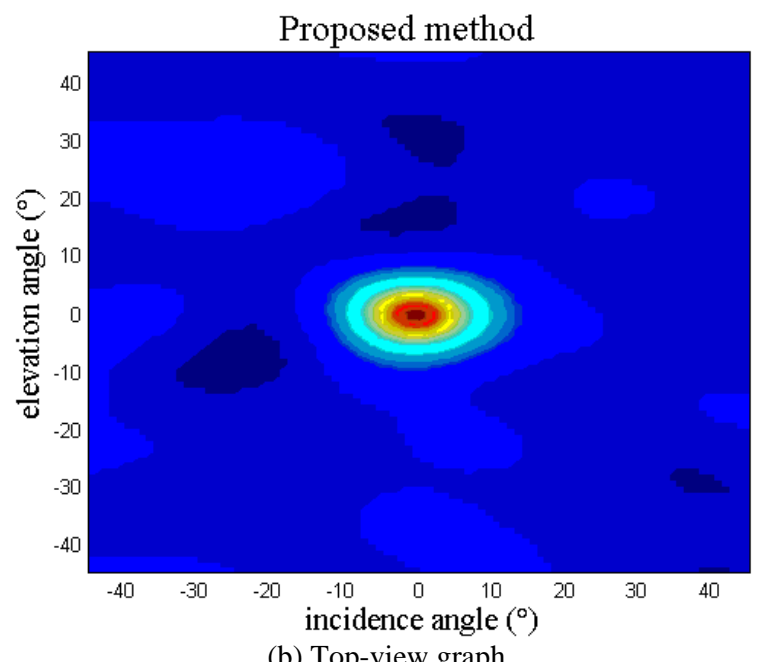

Figure 4. The spatial spectrum for VORB

\section{CONCLUSIONS}

In this paper, a robust Capon beamforming method based on a planar array has been proposed, and we have shown how to extend the original VORB algorithm to the case of a planar array. Computer simulation has demonstrated the excellent performance of the proposed method for the spatial spectrum estimation via a number of numerical examples.

\section{REFERENCES}

[1] Krim H, Viberg M. Two decades of array signal processing research: The parametric approach. IEEE Signal Process Mag, 1996, 13

[2] Naidu P S. Sensor Array Signal Processing. Boca Raton, Florida: CRC Press LLC, 2001

[3] Cox H, Zeskind R M, Owen M M. Robust adaptive beamforming[J]. IEEE Trans. Acoust., Speech, Signal Process., 1987:1365-1376.

[4] Jian Li, Petre Stoica, Zhisong Wang. On Robust Capon Beamforming and Diagonal Loading[J]. IEEE Transactions on signal processing, 2003, 51(7): 1702-1715.

[5] Vorobyov S A, Gershman A B, Luo Z Q. Robust Adaptive Beamforming Using Worst-Case Performance Optimization: A Solution to the Signal Mismatch Problem[J]. IEEE Transactions on Signal Processing, 2003, 51(2):313-324.

[6] Shi Jie, Yang De-Sen, and Shi Sheng-Guo. Robust localization and identification method of moving sound sources based on worst-case performance optimization. Acta Phys.Sin., 2011, 6(60):1-11.

[7] Song Hai-yan, Piao Sheng-chun, Xu Feng, and Xu Ya-jun. Robustness Analysis of Vector Optimization Adaptive Beamforming. Underwater Security and Technology, 2011, 1:153-157.

[8] Song Hai-yan, Piao Sheng-chun, and Qin Jin-ping. Performance Analysis of Robust Adaptive Beamforming Based on Vector Optimization. ACTA ELECTRONICA SINICA, 2012, 40(7):13511357.

[9] Sturm J F. Using SeDuMi 1.02, a MATLAB toolbox for optimization over symmetric cones. Optim Meth Softw, 1999, $11: 625-653 P$ 